\title{
Effects of dietary fibre intake on risk factors for cardiovascular disease in subjects at high risk
}

\author{
R Estruch, ${ }^{1,2}$ M A Martínez-González, ${ }^{3}$ D Corella, ${ }^{2,4} \mathrm{~J}$ Basora-Gallisá, ${ }^{5}$ V Ruiz-Gutiérrez, ${ }^{6}$ \\ M I Covas, ${ }^{2,7}$ M Fiol, ${ }^{2,8}$ E Gómez-Gracia, ${ }^{9}$ M C López-Sabater, ${ }^{10}$ R Escoda, ${ }^{1}$ M A Pena, ${ }^{11}$ \\ $\mathrm{J}$ Diez-Espino, ${ }^{3} \mathrm{C}$ Lahoz, ${ }^{12} \mathrm{~J}$ Lapetra, ${ }^{2,13} \mathrm{G}$ Sáez, ${ }^{14} \mathrm{E}$ Ros ${ }^{2,15}$ on behalf of the PREDIMED \\ Study Investigators
}

${ }^{1}$ Department of Internal

Medicine, Hospital Clinic, Institut d'Investigacions Biomèdiques

August Pi Sunyer (IDIBAPS),

Barcelona; ${ }^{2}$ Ciber Fisiopatologia de la Obesidad y Nutrición (CIBEROBN), Instituto de Salud Carlos III; ${ }^{3}$ Department of

Preventive Medicine and Public Health, School of Medicine-

Clinica Universitaria, University of Navarra; ${ }^{4}$ Department of Epidemiology, Preventive Medicine and Public Health, School of Medicine, University of Valencia; ${ }^{5}$ Direcció d'Atenció

Primària Reus-Tarragona, Institut Català de la Salut, Human Nutrition Unit, School of

Medicine, University Rovira i

Virgili, Reus (Tarragona);

${ }^{6}$ Instituto de la Grasa, Consejo

Superior de Investigaciones

Cientificas, Sevilla;

${ }^{7}$ Cardiovascular Epidemiology

Unit, Municipal Institut for

Medical Research (IMIM),

Barcelona; ${ }^{8}$ Institut Universitari

d'Investigació en Ciències de la

Salut (IUNICS), Palma de

Mallorca; ${ }^{9}$ Department of

Epidemiology, School of

Medicine, University of Malaga;

${ }^{10}$ Department of Nutrition and

Bromatology, School of

Pharmacy, Barcelona; ${ }^{11}$ Clinical

Trial Unit, Fundación LEIA-

Hospital Txangorritxu, Vitoria;

${ }^{12}$ Arteriosclerosis Unit, Hospital

Carlos III, Madrid; ${ }^{13}$ San Pablo

Health Center, Sevilla;

${ }^{14}$ Department of Biochemistry,

School of Medicine, University of Valencia; ${ }^{15}$ Lipid Clinic,

Endocrinology and Nutrition

Service, Institut d'Investigacions

Biomèdiques August Pi Sunyer

(IDIBAPS), Hospital Clinic,

Barcelona, Spain

Correspondence to:

Dr Ramon Estruch, Dept. of Internal Medicine, Hospital

Clinic, Villarroel, 170, 08036

Barcelona, Spain; restruch@

clinic.ub.es

Accepted 11 February 2009

Published Online First

15 March 2009

\begin{abstract}
Background: Epidemiological studies and feeding trials with supplements suggest that fibre intake is associated with a reduction in cardiovascular risk. However, the effects of changes in dietary fibre on risk factor levels have not been evaluated in free-living individuals. Thus, the effects of changes in dietary fibre intake on cardiovascular risk factors were assessed over 3 months in free-living high-risk subjects.
\end{abstract}

Methods: 772 high-risk subjects (age $69 \pm 5$ years) were assigned to a low-fat diet or two Mediterranean-style diets. All participants received behavioural and nutritional education, including recommendations for increasing the consumption of vegetables, fruits, and legumes. Changes in food and nutrient intake, body weight, blood pressure, lipid profiles, glucose control and inflammatory markers were evaluated.

Results: Most participants increased consumption of vegetable products, but the increase in dietary fibre exhibited wide between-subject variability (6-65 g/day). Body weight, waist circumference, and mean systolic and diastolic blood pressure decreased across quintiles of fibre intake $(p<0.005$; all). Reductions in fasting glucose and total cholesterol levels, and increments in $\mathrm{HDL}$ cholesterol were highest among participants in the upper $20 \%$ of fibre intake ( $p=0.04$ and 0.02 respectively).

Plasma concentrations of $\mathrm{C}$-reactive protein, but not those of inflammatory cytokines, decreased in parallel with increasing dietary fibre $(p=0.04)$. Significant reductions in LDL cholesterol were observed only among participants with the greatest increases in soluble fibre intake

$(p=0.04)$.

Conclusions: Increasing dietary fibre intake with natural foods is associated with reductions in classical and novel cardiovascular risk factors in a high-risk cohort.

The Mediterranean diet (Med-diet) has been widely considered as a model of healthy eating. This dietary pattern is characterised by a high consumption of non-refined grains, legumes, nuts, fruits and vegetables; relatively high intake of total fat, mainly derived from olive oil; moderate to high intake of fish and poultry; dairy products (usually as yogurt or cheese) in small amounts; low consumption of red meat and meat products; and moderate alcohol intake, usually in the form of red wine with meals. ${ }^{1}$ Thus, high consumption of fibre-rich foods is one of the characteristic features of the Med-diet.

Dietary fibre (DF), a mixture of chemicals in indigestible vegetable residues, ${ }^{2}$ has received much attention in nutritional epidemiology. Observational studies have consistently shown that DF intake is associated with reduced cardiovascular risk, including ischaemic heart disease ${ }^{3-6}$ and stroke, ${ }^{5-8}$ and lower risk of diabetes. ${ }^{9-11}$ Clinical trials have also suggested that DF supplementation has beneficial effects on risk factors, such as blood pressure, serum lipids, insulin sensitivity and diabetic metabolic control. ${ }^{12-16}$ However, no intervention study has evaluated the effects of DF on classical and novel risk factors in free-living persons at high cardiovascular risk.

To address this issue, the effects of 3-month changes in DF intake on cardiovascular risk factors were evaluated in a large sample of subjects at high risk for cardiovascular disease.

\section{METHODS}

\section{Study design}

The PREDIMED study (PREvención con DIeta MEDiterránea) is a large multi-centre, randomised 5 -year clinical trial aimed at assessing the effects of the Med-diet on the primary prevention of cardiovascular disease (CVD) in Spain (http:// www.predimed.org). Up to September 2008, this trial includes 6988 high-risk participants allocated to three interventions: Med-diet plus virgin oil, Med-diet plus nuts and control diet (low-fat diet). The main outcome for the whole trial is an aggregate of cardiovascular events (cardiovascular death, non-fatal myocardial infarction or non-fatal stroke). The Institutional Review Boards of the participating centres approved the study protocol. This trial has been registered with the International Standard Randomised Controlled Trial Number (ISRCTN) 35739639. The trial design has been described in detail elsewhere. ${ }^{17}$

The present study was designed as a short-term trial in a subset of participants, namely those entering the PREDIMED trial during the first 6 months of recruitment, to assess the effects of changes in dietary fibre intake on surrogate markers of atherosclerosis.

\section{Participants and recruitment}

Nine-hundred and thirty possible participants were evaluated. Eligible subjects were community-dwelling persons, aged 55-80 years for men and 60-80 years for women, who had either type 2 diabetes or three or more of the following $\mathrm{CHD}$ risk factors: current smoking, hypertension (blood pressure> 140/90 mmHg), LDL cholesterol $\geqslant 160 \mathrm{mg} / \mathrm{dl}$, low 
HDL cholesterol ( $\leqslant 40 \mathrm{mg} / \mathrm{dl}$ ), body mass index (BMI $\geqslant 25 \mathrm{~kg}$ / $\mathrm{m}^{2}$ ), or family history of premature CHD. Exclusion criteria were: previous history of cardiovascular disease, any severe chronic illness, illegal drug use or alcoholism, and low predicted likelihood of changing dietary habits. ${ }^{18}$

The primary care physicians based participants' eligibility on review of clinical records and a screening visit. Suitable candidates were invited to attend a screening visit. This included a face-to-face interview to inquire about medical conditions and risk factors related to eligibility. More than $95 \%$ of eligible candidates who met entry requirements signed an informed consent and agreed to return for the baseline visit.

\section{Baseline assessment and intervention}

A validated 137-item food frequency questionnaire (FFO), ${ }^{19}$ and the validated Spanish version ${ }^{20}$ of the Minnesota Leisure Time Physical Activity questionnaire were administered in the baseline assessment. For each food included in the FFQ, a standard portion size (representing the serving size of that item most frequently consumed currently in Spain) was specified. Dietary fibre intake was calculated by multiplying the frequency of consumption of each item by the fibre content of the specified serving, according to Spanish food composition tables. ${ }^{21}$ The intra-class correlation coefficient comparing DF intake by FFO with the average of four 3-day dietary records was 0.75. Anthropometric measurements, blood pressure, spot urine and fasting blood samples were obtained. All examinations were repeated at 3 months.

Trained dieticians were responsible for all aspects of the interventions and assisted participants in completing the FFO. ${ }^{22}$ Participants assigned to the control group received personal advice together with a leaflet with written recommendations to follow a low-fat diet. ${ }^{23}$ Participants in the two Med-diet intervention groups were given personalized advice for dietary changes directed to achieve a diet closest to the traditional Meddiet. ${ }^{22}$ Depending on group assignment, participants were given free virgin olive oil or free sachets of walnuts, hazelnuts and almonds. In the three groups, the general guidelines included positive recommendations for increased consumption of vegetables, fruits, legumes, fish and seafood, and white meats instead of red meats. Negative recommendations included limiting and/or eliminating presumed detrimental foods (red and processed meats, fat-rich dairy products, commercial pastries, snacks and sugar-sweetened beverages). Thus, all intervention diets coincided in the recommendation to increase fibre-rich foods, a reason why the three intervention groups are grouped as a single one for the comparisons of fibre intake and change in risk factor levels.

A 1-hour group session with up to 20 participants, with separate sessions for each intervention group, was scheduled after inclusion. Group sessions consisted of informative talks and provision of written material with descriptions of main foods, seasonal shopping lists, meal plans and cooking recipes. ${ }^{22}$

\section{Measurements}

Weight and height were measured with calibrated scales and a wall-mounted stadiometer respectively. Waist circumference was measured midway between the lowest rib and the iliac crest using an anthropometric tape. Trained personnel measured blood pressure in triplicate with a validated semi-automatic oscillometer (Omron HEM-705CP, The Netherlands).

Blood and urine samples were obtained after an overnight fast. Serum, EDTA- plasma, and urine samples were coded, shipped to central laboratories and stored at $-80^{\circ} \mathrm{C}$ until assay. The clinical investigators and laboratory technicians were blinded to intervention. Analyses determined by subject in frozen samples of whole serum or plasma as appropriate were: blood glucose by the glucose-oxidase method; serum insulin by radioimmunoassay; cholesterol and triglycerides by enzymatic procedures; HDL cholesterol after precipitation with phosphotungstic acid and magnesium chloride; soluble intercellular adhesion molecule-1 (ICAM-1), vascular cell adhesion molecule1 (VCAM-1) and interleukin-6 (IL-6) by standard ELISA assays; and high sensitivity C-reactive protein (CRP) by particleenhanced immunonephelometry.

\section{Statistical analyses}

For the comparison of changes in risk factors, the average of two baseline measurements was used as the baseline value and the average of the two 3-month measurements was used as the final variable. Values with a skewed distribution (CRP, VCAM-1, ICAM-1 and IL-6) were transformed to their natural logarithm for analyses. Those subjects whose energy intake, as derived from food frequency questionnaires, was outside pre-specified ranges (2510-14 $644 \mathrm{~kJ}$ for women and 3347-17 $573 \mathrm{~kJ}$ for men) were excluded from the calculations of energy and nutrient intake, as recommended in nutritional epidemiology. ${ }^{24}$ Multiple linear regression models were used to study the relationship between DF and changes in risk factors. Mean differences in the changes of these variables (post-trial minus pre-trial values) were compared by quintiles of change in DF intake. Adjustments were made for intervention group, age, gender, body weight, smoking, alcohol intake and physical activity. The treatment effects are expressed as average changes and $95 \%$ confidence intervals (CI).

\section{RESULTS}

Of the 930 eligible subjects, 158 were excluded for the following reasons: not meeting inclusion criteria $(n=86)$, not accepting to change their dietary habits $(n=18)$, excessive alcohol intake $(n=14)$, gastrointestinal disease $(n=4)$, food allergies $(n=3)$ or refusing to participate $(n=33)$. Table 1 shows the baseline characteristics of the 772 participants (348 men and 424 women) who entered the study. Most of them were overweight or obese $(90 \%)$, more than three-quarters had hypertension, one-quarter had family history of cardiovascular disease and one-fifth were smokers. There were only three withdrawals before study completion. The baseline data of these subjects were similar to the mean values for the overall group. Subsequent data refer only to the 769 participants who completed the study.

\section{Food, energy and nutrient intake}

Forty-eight participants were excluded from food, energy and nutrient calculations because they reported unrealistic energy intakes. ${ }^{21}$ Following the advice of the dieticians, most of the participants increased consumption of vegetables, legumes, fruits and fish, and decreased intake of meat and dairy products (table 2). Cereal intake also decreased due to reduced consumption of white bread, which is the main cereal food in the Spanish diet. A large increase in the consumption of nuts, one of the supplemental foods given, was observed, whereas the total amount of olive oil did not change because participants replaced customarily used refined olive oil by the virgin variety supplied. Alcohol intake increased slightly at the expense of wine, one of the components of the Med-diet recommended in moderation to 
Table 1 Baseline characteristics of the 772 subjects included in the study

\begin{tabular}{lc}
\hline Characteristics & Participants \\
\hline Age, mean (SD), years & $68.8(6.4)$ \\
Gender, men (\%) & $339(44)$ \\
Family history of CHD, no. (\%) & $178(23)$ \\
Current smokers, no. (\%) & $127(16)$ \\
Type 2 diabetes mellitus, no. (\%) & $421(54)$ \\
Hypertension, no (\%) & $605(78)$ \\
Dyslipidaemia, no. (\%) & $515(67)$ \\
Body mass index $>25 \mathrm{~kg} / \mathrm{m}^{2 *}$ & $29.9(4.2)$ \\
Overweight or obesity & $696(90)$ \\
Medications, no. (\%) & $340(44)$ \\
ACE inhibitors & $271(35)$ \\
Diuretics & $160(21)$ \\
Other antihypertensive agents & $326(42)$ \\
Statins & $51(6)$ \\
Other lipid-lowering agents & $61(8)$ \\
Insulin & $282(37)$ \\
Oral hypoglycaemic drugs & $138(18)$ \\
Aspirin or other antiplatelet drugs & \\
Occupation, no. (\%) & $179(23)$ \\
Unskilled & $288(37)$ \\
Skilled manual & $172(22)$ \\
Skilled non-manual & $133(17)$ \\
Directive and professional & \\
Education level, no. (\%) & $554(72)$ \\
Primary school & $126(16)$ \\
First degree high school & $91(12)$ \\
High school or university &
\end{tabular}

Abbreviations: CHD, coronary heart disease; ACE, angiotensin-converting enzyme.

${ }^{*}$ Calculated as weight in kilograms divided by the square of height in metres.

participants who were not abstainers at baseline. The results did not materially change when the participants whose energy consumption was out of range were included in the calculations.
Table 2 also shows 3-month changes in energy and nutrient intake. The reduction from baseline in reported energy intake was due to decreases in intake of carbohydrate and total fat, whereas protein intake increased. Increases in intake of monounsaturated fatty acids (MUFA) and polyunsaturated fatty acids (PUFA), including both vegetable and marine n-3 fatty acids, and a decrease in saturated fatty acid (SFA) intake occurred. Estimated energy expenditure from physical activity was similar at baseline and after 3 months.

\section{Fibre and vascular risk factors}

A significant increase in total and soluble DF intake was observed at the 3-month assessment (table 2). However, the consumption of DF exhibited wide between-subject variability. In fact, daily DF intake ranged from 6 to $65 \mathrm{~g}$ at the 3-month assessment. Because energy and DF intake increased in parallel, changes in DF intake categorised in quintiles were energyadjusted to assess associations with other variables (table 3). Demographic characteristics, adiposity and cardiovascular risk factors were similar across the range of DF intake. Predictably, increasing DF intake was associated directly with carbohydrate intake and inversely with intake of total fat, MUFA and SFA. There were no differences in alcohol intake or sedentariness by categories of DF intake.

Table 4 shows adjusted changes in risk factors according to quintiles of change in DF. The higher the increment in DF intake, the greater was the weight loss and the reduction in waist circumference and systolic and diastolic blood pressure. In addition, fasting glucose and total cholesterol levels decreased, and HDL cholesterol increased with increasing DF intake. Reductions in serum LDL cholesterol concentrations, but not in triglycerides, were not significantly higher for participants with greater increases in DF. The plasma levels of CRP, a systemic biomarker of inflammation, but not those of other inflammatory biomarkers, also decreased in parallel with increases in DF.

Table 2 Baseline levels and 3-month changes in the consumption of key food items, energy and nutrients

\begin{tabular}{|c|c|c|c|c|}
\hline Variables & Baseline & 3 months & Change, mean (95\% CI) & p Value \\
\hline \multicolumn{5}{|c|}{ Foods, daily consumption in grams } \\
\hline Fruits & $262(218)$ & $280(153)$ & $18.1(0.4$ to 35.7$)$ & 0.044 \\
\hline Vegetables & 357 (168) & $362(164)$ & $5.1(-8.0$ to 18.2$)$ & 0.443 \\
\hline Legumes & $14.3(9.3)$ & $19.5(21.5)$ & $5.2(3.6$ to 6.9$)$ & $<0.001$ \\
\hline Fish, seafood & $111(63)$ & $114(87)$ & $2.6(-3.6$ to 8.8$)$ & 0.414 \\
\hline Meat, meat products & $157(75)$ & $136(58)$ & $-20.6(-26.6$ to -15.2$)$ & $<0.001$ \\
\hline Cereals & $263(124)$ & $238(115)$ & $-24.8(-34.4$ to -15.2$)$ & $<0.001$ \\
\hline Dairy products & $403(228)$ & $387(226)$ & $-16.1(-32.1$ to -0.2$)$ & 0.047 \\
\hline Refined-mixed olive oil & $20.5(20.7)$ & $13.6(19.5)$ & $-6.9(-8.6$ to -5.1$)$ & $<0.001$ \\
\hline Virgin olive oil & $19.9(23.9)$ & $27.6(23.8)$ & 7.7 (5.9 to 9.4$)$ & $<0.001$ \\
\hline Total nuts & $11.0(16)$ & $22.1(23.5)$ & 11.1 (9.1 to 13.1$)$ & $<0.001$ \\
\hline Alcohol & $10.5(17.3)$ & $12.3(18.4)$ & $1.9(0.6$ to 3.1$)$ & 0.003 \\
\hline \multicolumn{5}{|l|}{ Energy and nutrients } \\
\hline Energy, kj/d & $2538(738)$ & $2393(638)$ & $-145(-200$ to -88$)$ & $<0.001$ \\
\hline Protein, \% En & $17.1(3.3)$ & $18.8(6.0)$ & 1.7 (1.2 to 2.3$)$ & $<0.001$ \\
\hline Carbohydrate, \% En & $41.9(7.7)$ & $41.0(7.70)$ & $-0.9(-1.5$ to -0.3$)$ & $<0.001$ \\
\hline Total fibre, $\mathrm{g} / \mathrm{d}$ & $21.1(7.6)$ & $22.2(7.9)$ & $1.1(0.4$ to 1.7$)$ & 0.001 \\
\hline Soluble fibre, $\mathrm{g} / \mathrm{d}$ & $5.5(2.1)$ & $5.9(2.0)$ & $0.4(0.2$ to 0.6$)$ & $<0.001$ \\
\hline Total fat, \% En & $38.2(7.3)$ & $37.2(9.1)$ & $-0.8(-1.5$ to -0.1$)$ & 0.020 \\
\hline MUFA & $18.8(4.6)$ & $19.3(4.6)$ & $0.5(0.1$ to 0.8$)$ & 0.009 \\
\hline PUFA & $6.8(2.2)$ & $7.2(3.2)$ & $1.0(0.8$ to 1.3$)$ & $<0.001$ \\
\hline SFA & $10.1(2.4)$ & $9.2(2.2)$ & $-0.8(-1.0$ to -0.7$)$ & $<0.001$ \\
\hline Alpha-linolenic acid, g/d & $1.5(0.9)$ & $1.9(1.4)$ & $0.4(0.3$ to 0.5$)$ & $<0.001$ \\
\hline Marine $\mathrm{n}-3$ fatty acids, $\mathrm{g} / \mathrm{d}$ & $0.8(0.6)$ & $0.9(0.9)$ & $0.1(0.0$ to 0.2$)$ & 0.009 \\
\hline
\end{tabular}

\footnotetext{
$\mathrm{Cl}$, Confidence Interval; MUFA, monounsaturated fatty acids; PUFA, polyunsaturated fatty acids; SFA, saturated fatty acids.
} 
Table 5 shows changes in cardiovascular risk factors between extreme quintiles of DF intake. The differences were statistically significant for body weight, waist circumference, systolic and diastolic blood pressure, fasting glucose and total cholesterol. When the same analysis was performed for changes in soluble DF intake, the results were similar, except for changes in LDL cholesterol, which were $-8.06 \mathrm{mg} / \mathrm{dl}$ (95\% CI 15.83 to $-2.87)$, significantly $(p=0.042)$ different between extreme quintiles. Changes of cardiovascular risk factors in quintile 5 versus quintile 1 of DF intake ranged from nearly $1 \mathrm{~kg}$ reduction in body weight to close to a $9 \mathrm{~mm}$ decrease in systolic blood pressure.

\section{DISCUSSION}

The hypothesis that the provision of increasing amounts of DF from natural foods might be instrumental in the effect of a Mediterranean-style diet to reduce the levels of cardiovascular risk factors was tested. The rationale underlying this substudy of the PREDIMED feeding trial was the available epidemiological and clinical trial evidence supporting the association of DF intake with beneficial effects on surrogate markers of cardiometabolic risk, including systemic inflammation. ${ }^{12}{ }^{14}{ }^{25-27}$ In fact, DF intake after a 3-month behavioural intervention to enhance the Med-diet was associated with significant reductions in body weight, waist circumference, systolic and diastolic blood pressure and fasting glucose, as well as a greater increase in HDL cholesterol. In addition, when changes in the consumption of soluble DF were specifically assessed, participants in the highest quintile also showed a significantly greater reduction in serum LDL cholesterol concentration than those in the lowest quintile.

Many epidemiological studies have evaluated the effects of DF on the risk of coronary heart disease..$^{3-6}$ In a pooled analysis of 10 prospective cohorts, ${ }^{28}$ each $10-\mathrm{g} /$ day increment of energyadjusted total DF was associated with a $14 \%$ decrease in risk of coronary events and a $27 \%$ decrease in risk of coronary death.
However, in the only randomised clinical trial designed to examine whether increased intake of DF reduces the risk of myocardial infarction, the DART study, ${ }^{29}$ patients with existing coronary heart disease advised to increase DF did not have lower reinfarction rates over a 2-year period. Furthermore, DF advice had no clear effect on coronary or all-cause mortality after longer follow-up. ${ }^{30}$ Most likely, as assessed in prospective studies, ${ }^{28}$ DF intake with the usual diet is a marker of healthy food choices with an overall cardiovascular benefit, whereas changing the diet to increase DF at late stages, when clinical consequences of atherosclerosis have developed, may not be protective, as has been shown in a similar situation for antioxidant supplements as opposed to the antioxidant content of the habitual diet. ${ }^{31}$ Indeed, a plant-based diet is rich in both fibre and antioxidants. At any rate, increasing DF during 3 months by older individuals at high cardiovascular risk has measurable beneficial effects on several risk factors.

Reduced adiposity, particularly in the highest category of DF intake (tables 4 and 5), was observed in the present study. Large prospective studies have reported that consumption of DF is inversely associated with weight. ${ }^{32-34}$ A high intake of DF may assist weight loss because of the incomplete digestion and absorption of energy from this type of carbohydrate and the bulky nature of high-fibre foods, with increased demands on chewing and subsequent distension and delayed emptying of the stomach, promoting satiety and thus curtailing energy intake. ${ }^{25} 2635$ The present results further support a beneficial role of fibre-rich diets on weight control.

Increasing DF intake was associated with significant blood pressure lowering in those mostly hypertensive participants. Consistently, large prospective studies have reported that increased DF was associated with lower risk of hypertension ${ }^{36}$ or lower self-reported blood pressure. ${ }^{37}$ A meta-analysis of clinical studies of fibre supplementation ${ }^{12}$ also supports an inverse association between fibre and blood pressure. Accordingly, an increase in DF has been recommended by the

Table 3 Subjects' characteristics and energy and nutrient intake according to quintiles of energy-adjusted dietary fibre intake at 3 months

\begin{tabular}{|c|c|c|c|c|c|c|}
\hline \multirow[b]{2}{*}{ Variables } & \multicolumn{5}{|c|}{ Quintiles of energy-adjusted total fibre intake at baseline } & \multirow[b]{2}{*}{ p Value } \\
\hline & 01 & 02 & 03 & 04 & 05 & \\
\hline $\mathrm{N}$ & 144 & 146 & 143 & 143 & 145 & \\
\hline Dietary fibre intake, $\mathrm{g} / \mathrm{d}$ (median) & $\leqslant 16(14)$ & $16.1-19.9(18)$ & $20.0-21.9(21)$ & $22.0-25.9(23)$ & $\geqslant 26.0(29)$ & \\
\hline Age, years & $68.7(5.6)$ & $69.3(6.0)$ & $68.4(6.5)$ & $68.9(6.0)$ & $69.2(7.6)$ & 0.667 \\
\hline Sex, men & 60 & 77 & 62 & 64 & 64 & 0.609 \\
\hline Smokers, n (\%) & $27(19)$ & $26(18)$ & $24(17)$ & $17(12)$ & $28(19)$ & 0.733 \\
\hline Diabetes, n (\%) & $79(55)$ & $95(65)$ & $77(54)$ & $75(52)$ & $85(59)$ & 0.470 \\
\hline Hypertension, n (\%) & $125(87)$ & $130(89)$ & $111(78)$ & $108(76)$ & $112(77)$ & 0.294 \\
\hline Dyslipidaemia, n (\%) & $105(73)$ & $100(69)$ & $88(62)$ & $107(75)$ & $94(65)$ & 0.126 \\
\hline Body mass index, $\mathrm{kg} / \mathrm{m}^{2}$ & $29.6(4.0)$ & $30.0(4.5)$ & $30.0(4.5)$ & $29.7(4.0)$ & $29.3(3.9)$ & 0.608 \\
\hline Waist circumference, $\mathrm{cm}$ & $101(10)$ & $101(12)$ & $100(11)$ & $99(13)$ & $98(10)$ & 0.174 \\
\hline Energy, kcal/d & $2059(590)$ & $2324(520)$ & $2538(598)$ & $2824(724)$ & $3065(886)$ & $<0.001$ \\
\hline Protein, \% En & $17.6(3.8)$ & $17.2(2.9)$ & $16.9(3.4)$ & $16.6(2.9)$ & $16.8(2.8)$ & 0.043 \\
\hline Carbohydrate,\% En & $40.6(8.5)$ & $40.3(7.6)$ & $41.5(6.9)$ & $43.7(6.8)$ & $45.2(6.8)$ & $<0.001$ \\
\hline Fat, \% En & $38.3(7.9)$ & $39.7(7.3)$ & $38.9(7.2)$ & $37.4(6.1)$ & $35.8(6.1)$ & $<0.001$ \\
\hline MUFA & $18.8(5.0)$ & $19.8(2.5)$ & $19.3(4.6)$ & $18.3(4.1)$ & $16.9(3.8)$ & $<0.001$ \\
\hline PUFA & $5.8(2.1)$ & $6.2(1.8)$ & $6.2(2.2)$ & $6.0(1.8)$ & $6.3(2.7)$ & 0.215 \\
\hline SFA & $10.7(2.7)$ & $10.4(2.5)$ & $10.1(2.2)$ & $9.8(2.1)$ & $9.6(2.4)$ & $<0.001$ \\
\hline Virgin olive oil, g/d & $13.8(20.9)$ & $18.8(22.8)$ & $19.5(23.1)$ & $24.1(25.4)$ & $20.0(23.4)$ & 0.010 \\
\hline Alcohol, g/d & $11.8(22.7)$ & $9.9(16.7)$ & $10.8(16.8)$ & $9.1(13.5)$ & $9.2(11.7)$ & 0.754 \\
\hline Low physical activity, $\mathrm{n}(\%) \dagger$ & $47(34)$ & $45(32)$ & $46(33)$ & $35(25)$ & $32(23)$ & 0.155 \\
\hline
\end{tabular}

Abbreviations as in table 2.

Values are means (standard deviations) unless otherwise stated.

${ }^{*} \mathrm{p}$ Value for linear trend by ANOVA or chi-square test.

†Lowest tertile. 
Table 4 Three-month changes in risk factors by quintiles of change in dietary fibre intake

\begin{tabular}{|c|c|c|c|c|c|c|c|}
\hline \multirow[b]{2}{*}{ Variable changes } & \multicolumn{7}{|c|}{ Quintiles of change in dietary fibre intake } \\
\hline & 01 & 02 & 03 & 04 & 05 & p Value* & p Value** \\
\hline Fibre intake, range $(\mathrm{g} / \mathrm{d})$ & $<-2.5$ & -2.5 to 0.2 & 0.2 to 1.8 & 1.8 to 5.3 & $>5.3$ & & \\
\hline $\mathrm{N}$ & 122 & 123 & 123 & 122 & 123 & & \\
\hline Weight, kg & 0.02 & -0.19 & -0.23 & -0.38 & -0.90 & $<0.001$ & 0.001 \\
\hline Waist circumference, $\mathrm{cm}$ & 0.74 & -0.17 & -0.83 & -1.27 & -1.90 & $<0.001$ & $<0.001$ \\
\hline Fasting glucose, mg/dl & 5.72 & 1.84 & -3.56 & -1.65 & -7.67 & 0.05 & 0.04 \\
\hline Fasting insulin, pmol/l & -0.53 & 0.51 & -0.68 & -0.52 & -0.77 & 0.81 & 0.76 \\
\hline Total cholesterol, mg/dl & 0.94 & -2.75 & 0.95 & -5.52 & -8.78 & 0.14 & 0.19 \\
\hline LDL cholesterol, mg/dl & -1.66 & -2.15 & -3.77 & -5.09 & -8.63 & 0.14 & 0.14 \\
\hline HDL cholesterol, mg/dl & 1.29 & 0.81 & 1.46 & 1.51 & 2.32 & 0.07 & 0.02 \\
\hline Triglycerides, mg/dl & -5.09 & -4.20 & 0.70 & -2.23 & -3.62 & 0.80 & 0.70 \\
\hline
\end{tabular}

BP, blood pressure; ICAM, intercellular adhesion molecule; VCAM, vascular cell adhesion molecule.

${ }^{*} \mathrm{p}$ Value for linear trend adjusted for age, gender and energy intake.

${ }^{* *}$ Additionally adjusted for intervention group and baseline body mass index, smoking, alcohol intake and physical activity.

World Health Organization as a safe and practical approach to cardiovascular risk reduction in hypertensive populations. ${ }^{38}$

Participants who increased DF intake also showed a reduction in fasting glucose levels, extending previous observations of the favourable effects of a diet with abundant fibre-rich foods, particularly whole grain, bran and germ intake, on the risk of type 2 diabetes. ${ }^{9-11} 3940$ Dietary fibre reduces the glycaemic index of foods, thereby attenuating insulin responses and enhancing insulin sensitivity, ${ }^{41}{ }^{42}$ to which associated weight loss is likely to contribute.

Structural fibre is insoluble, whereas natural gel-forming fibres are soluble, ${ }^{2}$ and increasing consumption of this particular variety of DF has a modest cholesterol-lowering effect that is attributable to interference with intestinal bile acid absorption and enhancement of faecal cholesterol excretion, although other mechanisms can be operative. ${ }^{42}$ Thus, increasing soluble fibre intake by $1 \mathrm{~g} /$ day is associated with a mean reduction in serum LDL cholesterol level of about $2 \mathrm{mg} / \mathrm{dl}(0.052 \mathrm{mmol} / \mathrm{l})$, according to a meta-analysis of randomised trials of soluble fibre supplementation..$^{13}$ In support of these evidences, the present study shows a significant $-8.06 \mathrm{mg} / \mathrm{dl} \mathrm{LDL}$ cholesterol decrease between extreme quintiles of soluble fibre intake.

As atherosclerosis is considered a low-grade inflammatory disease, $^{43}$ the relationship between DF intake and serum inflammatory markers has also been evaluated in epidemiological and clinical studies, as reviewed. ${ }^{25-27}$ Results from recent epidemiological studies have consistently shown an inverse association between DF intake and plasma CRP levels. ${ }^{44-47}$ In a recent study, both increasing fibre intake by about $30 \mathrm{~g}$ /day from a diet naturally rich in fibre or from a supplement reduced levels of CRP. ${ }^{48}$ Thus, it was not surprising that a nearly $1 \mathrm{mg} / \mathrm{L}$ reduction in CRP levels was observed in the upper quintile of energy-adjusted DF intake ( $\leqslant 26 \mathrm{~g} /$ day), after adjustment for sex, age, BMI and relevant lifestyle characteristics. However, no changes were observed in other inflammatory biomarkers. The mechanisms of change in CRP levels as a result of DF intake are still largely unknown. Possibilities include DF slowing absorption of glucose, fibre-rich meal modulation of cytokine responses blunting oxidative stress and inflammation, and production of anti-inflammatory cytokines by gut flora exposed to fibre. ${ }^{26}{ }^{27}$

The present study has limitations. As no biomarker of DF intake is available, FFO data were the only source of information on food consumption, including DF. Food frequency questionnaires are known to contain measurement errors, a reason why energy intake was included as a covariate in the models to achieve the equivalent of an isoenergetic diet and thus overcome this problem. At any rate, measurement error

Table 5 Mean changes (95\% confidence intervals) and mean differences (95\% confidence intervals) in changes in adiposity, blood pressure and other risk factors between the lowest (01) and the highest (05) quintile of changes in total dietary fibre intake

\begin{tabular}{|c|c|c|c|c|}
\hline \multirow[b]{2}{*}{ Variable changes } & \multicolumn{3}{|c|}{ Extremes of changes in dietary fibre intake } & \multirow[b]{2}{*}{ p Value** } \\
\hline & 01 & 05 & Change 05 versus $01 *$ & \\
\hline Weight, kg & $0.02(-0.36$ to 0.32$)$ & $-0.90(-1.40$ to -0.42$)$ & $-0.92(-1.52$ to -0.33$)$ & 0.002 \\
\hline Waist circumference, $\mathrm{cm}$ & $0.74(-0.20$ to 1.68$)$ & $-1.90(-3.18$ to -0.60$)$ & $-2.63(-4.20$ to -1.07$)$ & 0.001 \\
\hline Systolic blood pressure, mmHg & $-0.19(-3.41$ to 3.03$)$ & $-9.08(-12.21$ to -5.95$)$ & $-8.89(-13.37$ to -4.41$)$ & $<0.001$ \\
\hline Diastolic blood pressure, $\mathrm{mmHg}$ & $-1.25(-2.70$ to 0.21$)$ & $-4.76(-6.46$ to -3.08$)$ & $-3.52(-5.73$ to -1.31$)$ & 0.002 \\
\hline Fasting glucose, mg/dl & $5.72(0.64$ to 10.79$)$ & $-7.67(-11.84$ to -3.50$)$ & $-13.39(-19.86$ to -6.93$)$ & $<0.001$ \\
\hline Total cholesterol, mg/dl & $0.94(-5.24$ to 7.12$)$ & $-8.78(-14.30$ to -3.27$)$ & $-9.73(-17.96$ to -1.49$)$ & 0.021 \\
\hline LDL cholesterol, mg/dl & $-1.66(-6.96$ to 3.64$)$ & $-8.63(-14.26$ to -3.00$)$ & $-7.90(-14.96$ to 0.72$)$ & 0.075 \\
\hline HDL cholesterol, mg/dl & $1.29(0.08$ to 2.52$)$ & $2.32(1.08$ to 3.57$)$ & $1.03(-0.70$ to 2.76$)$ & 0.242 \\
\hline C-reactive protein, mg/l & $-0.02(-0.51$ to 0.48$)$ & $-1.01(-1.53$ to -0.49$)$ & $-1.08(-1.80$ to -0.48$)$ & 0.004 \\
\hline
\end{tabular}

\footnotetext{
${ }^{*}$ Differences between baseline and 3-month follow-up evaluation.
} 
most likely would have introduced non-differential misclassification, and the implications for the results of this error would have been to bias the estimates towards the null. The duration of follow-up lasting only 3 months cannot be considered a major limitation, because the effects of dietary interventions on risk factors do not need a long induction period. ${ }^{49}$ The study also has strengths, such as reproducing real-life conditions with home-prepared foods in free-living individuals, as in usual clinical practice. Other strengths are the high response and low drop-out rates, which can be partly explained because participants belonged to a Mediterranean culture in which people are accustomed to using olive oil and nuts and enjoy eating fibrerich vegetables, fruits and legumes, and the supplemental foods were provided at no cost.

In summary, the results of this study show that an increase in DF intake achieved with natural foods in the setting of a Mediterranean-style diet can reduce cardiovascular risk factors. Except for subjects in the highest $20 \%$ of DF intake, the effects on each individual risk factor were modest but, taken together, they suggest that increasing DF consumption may be instrumental in modifying the population cardiovascular risk profile, therefore preventing or delaying future cardiovascular events.

Acknowledgements: We thank the participants for their enthusiastic collaboration and all the PREDIMED personnel for excellent assistance with all aspects of the trial. The authors are grateful to grant support by the Spanish Minister of Health (RTIC G03/ 140 and RD06/0045), the FIS 070473, Centro Nacional de Investigaciones Cardiovasculares (CNICO6) and MADAUS Pharm for their collaboration in the study. Emili Corbella provided expert assistance with statistical analyses. CIBEROBN is an initiative of Instituto de Salud Carlos III, Spain.

Funding: Grant support by the Spanish Minister of Health (RTIC G03/140 and RD06/ 0045), the FIS 070473, Centro Nacional de Investigaciones Cardiovasculares (CNICO6) and MADAUS Pharm.

\section{What is already known on this subject}

- Epidemiological studies have suggested that dietary fibre intake is associated with reduced incidence of ischaemic heart disease, stroke and diabetes.

- Clinical trials have also pointed out that diets with fibre supplementation may reduce blood pressure, improve lipid profile and increase insulin sensitivity.

- No intervention studies have evaluated the effects of dietary fibre on classical and novel risk factors in free-living subjects at high cardiovascular risk.

\section{What this study adds}

- The increase of dietary fibre in an intervention aimed to upgrade consumption of fruits, vegetables and legumes in the setting of a Mediterranean-style diet was associated with reductions in cardiovascular risk factors, such as body weight, waist circumference, systolic and diastolic blood pressures, serum glucose and C-reactive protein.

- Reductions in total cholesterol and LDL cholesterol were observed only among participants with greater increases in soluble fibre intake.

- These beneficial effects on surrogate markers of cardiovascular risk add biological plausibility to the epidemiological evidence supporting a protective effect of dietary fibre intake on the risk for cardiovascular diseases.
Competing interests: None.

Ethics approval: The Institutional Review Boards of the participating centres approved the study protocol (eg Hospital Clinic, Barcelona, Spain, as the coordinating centre).

\section{REFERENCES}

1. Willett WC, Sacks FM, Trichopoulou A, et al. Mediterranean diet pyramid: a cultural model for healthy eating. Am J Clin Nutr 1995;61(Suppl 6):1402-6.

2. Panel of Definition of Dietary Fiber, Standing Committee on the Scientific Evaluation of Dietary Referent Intakes, Food and Nutrition Board, Institute of Medicine. Dietary Reference Intakes: Proposed Definition of Dietary Fiber. Washington, DC: National Academies Press, 2001.

3. Rimm EB, Ascherio A, Giovannucci $E$, et al. Vegetable, fruit and cereal fiber intake and risk of coronary heart disease among women. JAMA 1996;275:447-51.

4. Todd S, Woodward M, Tunstall-Pedoe $\mathrm{H}$, et al. Dietary antioxidant vitamins and fibe in the etiology of cardiovascular disease and all-causes-mortality: results from the Scottish Heart Health Study. Am J Epidemiol 1999;150:1073-80.

5. Liu S, Buring JE, Sesso HD, et al. A prospective study of dietary fiber intake and risk of cardiovascular disease among women. J Am Coll Cardiol 2002;39:49-56.

6. Mozaffarian D, Kumanyika SK, Lemaitre RN, et al. Cereal, fruit and vegetable fiber intake and the risk of cardiovascular disease in elderly individuals. JAMA 2003;289:1659-66.

7. Ascherio A, Rimm EB, Hernan MA, et al. Intake of potassium, magnesium, calcium and fiber and risk of stroke among US men. Circulation 1998;98:1198-204.

8. Oh K, Hu FB, Cho E, et al. Carbohydrate intake, glycemic index, glycemic load, and dietary fiber in relation to risk of stroke in women. Am J Epidemiol 2005;161:161-9.

9. Salmeron J, Manson JE, Stampfer MJ, et al. Dietary fiber, glycemic load, and risk of non-insulin-dependent diabetes mellitus in women. JAMA 1997;277:472-7.

10. Meyer KA, Kushi LH, Jacobs DR Jr, et al. Carbohydrates, dietary fiber, and incident type 2 diabetes in older women. Am J Clin Nutr 2000:71:921-30.

11. Liu S. Whole-grain foods, dietary fiber, and type 2 diabetes: searching for a kernel of truth. Am J Clin Nutr 2003;77:527-9.

12. Streppel MT, Arends LR, van't Veer $P$, et al. Dietary fiber and blood pressure. A meta-analysis of randomized placebo-controlled trials. Arch Intern Med 2005; 165:150-6.

13. Brown L, Rosner B, Willett WW, et al. Cholesterol lowering effects of dietary fiber: a meta-analysis. Am J Clin Nutr 1999:69:30-42.

14. Anderson JW. Dietary fiber prevents carbohydrate-induced hypertriglyceridemia. Curr Atheroscler Rep 2000;2:536-41.

15. Chandalia M, Garg A, Lutjohann D, et al. Beneficial effects of high dietary fiber intake in patients with type 2 diabetes mellitus. N Engl J Med 2000;342:1392-8.

16. Ludwig DS, Pereira MA Kroenke $\mathrm{CH}$, et al. Dietary fiber, weight gain, and cardiovascular disease risk factors in younger adults. JAMA 1999;282:1539-46.

17. Estruch R, Martínez González MA, Corella D, et al. Effects on a Mediterranean-style diet on cardiovascular risk factors. A randomized trial. Ann Intern Med 2006;145:1-11.

18. Nigg CR, Burbank PM, Padula C, et al. Stages of change across ten health risk behaviors for older adults. Gerontologist 1999:39:473-82.

19. Martín-Moreno JM, Boyle P, Gorgojo L, et al. Development and validation of a food frequency questionnaire in Spain. Int J Epidemiol 1993;22:512-9.

20. Elosua R, Marrugat J, Molina L, et al. Validation of the Minnesota Leisure Time Physical Activity Questionnaire in Spanish Men. Am J Epidemiol 1994;139:1197-209.

21. Mataix J. Tabla de Composición de Alimentos Españoles, $4^{\text {th }}$ edn. Granada, Spain Universidad de Granada, 2003.

22. Zazpe I, Sanchez-Tainta A, Estruch $\mathrm{R}$, et al. A large randomized individual and group intervention conducted by registered dietitians increased adherence to Mediterranean-type: the PREDIMED study. J Am Diet Assoc 2008;108:1134-44.

23. Krauss RM, Eckel RH, Howard B, et al. AHA dietary guidelines. Revision 2000: A statement for healthcare professionals from nutrition committee of the American Heart Association. Circulation 2000:102:2284-99.

24. Willett WC. Issues in analysis and presentation of dietary data. In: Willett WC, ed Nutritional Epidemiology. New York: Oxford University Press, 1998:321-45.

25. Bulló M, Casas-Agustench P, Amigó-Correig P, et al. Inflammation, obesity and comorbidities: the role of diet. Public Health Nutr 2007;10:1164-72.

26. Weickert MO, Pfeiffer AFH. Metabolic effects of dietary fiber consumption and prevention of diabetes. J Nutr 2008;138:439-42.

27. O'Keefe JH, Gheewala NM, O'Keefe JO. Dietary strategies for improving postprandial glucose, lipids, inflammation, and cardiovascular health. J Am Coll Cardiol 2008;51:249-55.

28. Pereira MA, O'Reilly E, Augustsson K, et al. Dietary fiber and risk of coronary heart disease: a pooled analysis of cohort studies. Arch Intern Med 2004;164:370-6.

29. Burr ML, Fehily AM, Gilbert JF, et al. Effects of changes in fat, fish, and fibre intakes on death and myocardial reinfarction: diet and reinfarction trial (DART). Lancet 1989;2:757-61.

30. Ness AR, Hughes J, Elwood PC, et al. The long term effect of dietary advice in men with coronary disease: follow-up of the Diet and Reinfarction trial (DART). Eur J Clin Nutr 2002:56:512-8

31. Vivekananthan DP, Penn MS, Sapp SK, et al. Use of antioxidant vitamins for the prevention of cardiovascular disease: meta-analysis of randomised trials. Lancet 2003:361:2017-23. 
32. Liu S, Willett WC, Manson JE, et al. Relation between changes in intakes of dietary fiber and grain products and changes in weight and development of obesity among middle-aged women. Am J Clin Nutr 2003;78:920-7.

33. Koh-Banerjee $\mathbf{P}$, Franz M, Sampson L, et al. Changes in whole-grain, bran, and cereal fiber consumption in relation to 8-y weight gain among men. Am J Clin Nutr 2004;80:1237-45.

34. Bes-Restrollo M, Martínez-González MA, Sánchez-Villegas A, et al. Association of fiber intake and fruit/vegetable consumption with weight gain in a Mediterranean population. Nutrition 2006;22:504-11.

35. van Dam RM, Seidell JC. Carbohydrate intake and obesity. Eur J Clin Nutr 2007:61(Suppl 1):S75-99.

36. Ascherio A, Rimm EB, Giovannucci EL, et al. A prospective study of nutritional factors and hypertension among US men. Circulation 1992;86:1475-84.

37. Ascherio A, Hennekens C, Willett WC, et al. Prospective study of nutritional factors, blood pressure, and hypertension among US women. Hypertension 1996:27:1065-72.

38. WHO. Population nutrient intake goals for preventing diet-related chronic diseases. In: Diet, nutrition and the prevention of chronic diseases.WHO Technical report Series 2003;916:46-83.

39. Montonen J, Knekt $\mathrm{P}$, Jarvinen $\mathrm{R}$, et al. Whole grain and fiber intake and the incidence of type 2 diabetes. Am J Clin Nutr 2003;77:622-9.

40. van Dam RM, Hu FB, Rosenberg $L$, et al. Dietary calcium and magnesium, major food sources, and risk of type 2 diabetes in U.S. black women. Diabetes Care 2006;29:2238-43.
41. Bessesen DH. The role of carbohydrates in insulin resistance. J Nutr 2001;131:2782S-6S.

42. Jenkins DJ, Kendall CW, Axelsen M, et al. Viscous and nonviscous fibres, nonabsorbable and low glycaemic index carbohydrates, blood lipids and coronary heart disease. Curr Opin Lipidol 2000;11:49-56.

43. Hanson GK. Inflammation, atherosclerosis, and coronary artery disease. N Eng/ J Med 2005;352:1685-95

44. Ajani UA, Ford ES, Mokdad AH. Dietary fiber and C-reactive protein: findings from National Health and Nutrition Examination Survey data. J Nutr 2004;134:1181-5.

45. Ma Y, Griffith JA, Chasan-Taber L, et al. Association between dietary fiber and serum C-reactive protein. Am J Clin Nutr 2006;83:760-6.

46. Oi L, van Dam RM, Liu S, et al. Whole-grain, bran, and cereal fiber intakes and markers of systemic inflammation in diabetic women. Diabetes Care 2006;29:207-11.

47. Ma Y, Hébert JR, Li W, et al. Association between dietary fiber and markers of systemic inflammation in the Women's Health Initiative Observational Study. Nutrition 2008;24:941-9.

48. King DE, Egan BM, Woolson RF, et al. Effect of a high-fiber diet vs a fiber-supplemented diet on C-reactive protein level. Arch Intern Med 2007; 167:502-6.

49. Sacks FM, Svetkey LP, Vollmer WM, et al. Effects on blood pressure of reduced dietary sodium and the dietary approaches to stop hypertension (DASH) diet. N Engl J Med 2001;344:3-8 